

REVISÃO BIBLIOGRÁFICA

\title{
Comprometimento da qualidade de vida dos cuidadores de pacientes em tratamento paliativo
}

\author{
Commitment to the quality of life of caregivers of patients under palliative treatment \\ Compromiso com lacalidad de vida de los cuidadores de pacientes em tratamento \\ paliativo
}

\begin{abstract}
Samila Alves Garcia ${ }^{1 *}$, Mônica Isaura Corrêa², Aline Mara Botin Sudário¹, Anna Luíza Dalmaschio Barros $^{1}$, Celmara Caldeira Gomes Moura ${ }^{1}$, Juliana Gonçalves Araujo ${ }^{1}$, Letícia Pires de Oliveira ${ }^{1}$, Mariely Medeiros Soares ${ }^{1}$, Pedro Antonio Rodrigues Dias ${ }^{1}$, Tatiane Costa Pereira ${ }^{1}$.
\end{abstract}

\begin{abstract}
Resumo: Este artigo buscou abordar a qualidade de vida dos familiares/cuidadores de pacientes que recebem assistência em domicílio, considerando que a prestação de cuidados nesse modelo, exige muito tempo da vida desses profissionais e/ou familiares envolvidos nesse processo. Abordando também os sentimentos envolvidos nesse delicado processo, tanto de quem cuida, quanto de quem recebe esse cuidado, e como passam pelo processo do luto e dos desafios dessa jornada. Como resultado foi observado que o envolvimento do cuidador, enquanto membro da família, por dedicar-se por longos períodos ao enfermo, tem áreas da sua vida comprometida, podendo acarretar sobrecarga física, emocional como problemas psicológicos e/ou dificuldades financeiras. Assim sendo, a prevenção à saúde mental é a melhor estratégia para evitar que esses profissionais desenvolvam sintomas depressivos, advindos desse acúmulo de funções e carga emocional vivida, e em decorrência tenham sua qualidade de vida comprometida. Nos mostrando a necessidade da integração de uma equipe multiprofissional no auxílio ao cuidador e paciente, proporcionando uma abordagem humanizada de forma ampla e respeitando os valores e hábitos envolvidos em todo processo de cuidado.
\end{abstract}

Palavras-chave: Cuidador, Cuidados paliativos, Qualidade de vida.

\begin{abstract}
This article sought to address the quality of life of caregivers of patients who receive home care, considering the provision of care in this model, demands a lot of time in the life of these professionals or family members involved in this process. Also addressing the feelings involved in this process, both of those who give care, as well as those who receive this care, and how they go through the process of mourning and the challenges of this journey. As a result, it was observed that the caregiver's involvement, act as a family member, by dedicating him/herself for long periods of time to the patient, he/she has areas of his/her life compromised, which can lead to emotional overload, physical overload, psychological problems or financial difficulties. Therefore, preparedness of mental health is the best strategy to prevent these professionals from developing depressive symptoms, arising from this accumulation of duties and emotional burden experienced, and as a result their quality of life is compromised. Showing us the need to integrate a multi-professional team in helping caregivers and patients, providing a broad humanized approach and respecting the values, and being obliged in every care process.
\end{abstract}

Keywords: Caregiver, Palliative care, Quality of life.

${ }^{1}$ Centro Universitário de Caratinga (UNEC), Caratinga - MG. *E-mail: samilaalvesgarcia@gmail.com

${ }^{2}$ Instituto Metropolitano de Ensino Superior (IMES UNIVAÇO), Ipatinga - MG. 
Resumen: Este artículo buscó abordar la calidad de vida de los cuidadores de pacientes que reciben atención domiciliaria, considerando que la prestación de cuidados en este modelo, demanda mucho tiempo en la vida de estos profesionales o familiares involucrados en este proceso. Abordar también los sentimientos involucrados en este proceso, tanto de los que brindan cuidados, como de quienes reciben este cuidado, y cómo atraviesan el proceso de duelo y los desafíos de este camino. Como resultado, se observó que la implicación del cuidador, actuar como un miembro de la familia, al dedicarse por largos períodos de tiempo al paciente, tiene áreas de su vida comprometidas, lo que puede llevar a una sobrecarga emocional, sobrecarga física, problemas psicológicos o dificultades económicas. Por tanto, la preparación de la salud mental es la mejor estrategia para evitar que estos profesionales desarrollen síntomas depresivos, derivados de esta acumulación de deberes y carga emocional experimentada, y como consecuencia su calidad de vida se vea comprometida.Mostrándonos la necesidad de la integración de un equipo multiprofesional en la ayuda al cuidador y al paciente, brindando un amplio enfoque humanizado y respetando los valores y hábitos involucrados en todo el proceso asistencial.

Palabras clave: Cuidador, Cuidados paliativos, Calidad de vida.

\section{INTRODUÇÃO}

O termo qualidade de vida vem sendo objeto de pesquisa em diversos campos de estudo, em várias áreas de atuação. Considera-se que a qualidade de vida seja formada por um conjunto de fatores, como a percepção do próprio indivíduo sobre o andamento de sua vida, embasado nos valores e na cultura em que está inserido, bem como suas expectativas, realizações, objetivos e preocupações. Preocupar-se com a qualidade de vida é trazer a valorização de aspectos que envolvem a vida do profissional como um todo, como aparecimento e controle de sintomas de adoecimento, redução da taxa de mortalidade e aumento da expectativa de vida. É fundamental que sejam destinados recursos para a medicina curativa, mas, sobretudo, a medicina preventiva (ROCHA EM e SOUZA A, 2017).

Os cuidadores de pacientes que necessitam de cuidados paliativos em domicílio podem experimentar sentimentos paradoxais e conflituosos, não somente pela responsabilidade com o trabalho, o estresse diário, mas também com o desempenho de funções que não eram regularmente desempenhadas. Estes sentimentos podem exigir muito dessas pessoas tanto no aspecto físico, social, psíquico, econômico e intelectual. Ao mesmo tempo em que o cuidador se sente responsável pelo doente e nutre por ele algum tipo de afeto, pode sentir, devido à sua sobrecarga, algum sentimento contraditório, como raiva e depressão. Quando o cuidador é membro da família, este abdica de suas atividades rotineiras, afastando-se de sua vida social e às vezes profissional, trazendo grande prejuízo à sua qualidade de vida (PRUDENTE COM, et al., 2017).

Os cuidadores não oferecem somente cuidados de saúde e higiene, mas suporte para a vida diária, apoio social, que é fundamental para o paciente. Contudo, essa relação tão próxima afeta diretamente a qualidade de vida do cuidador, que lida com a vida, fragilidade do corpo, possibilidades de adoecimento e morte. Estudos apontam que cuidadores de membros da família possuem $63 \%$ mais chance de morrer em 04 anos de cuidados do que os demais membros da família (LACERDA MS, et al., 2019).

Diante desse contexto, o objetivo deste trabalho foi revisar através de uma análise como a assistência domiciliar de cuidados paliativos pode afetar a qualidade de vida de seus cuidadores.

\section{REVISÃO BIBLIOGRÁFICA}

\section{O Paciente e o Cuidador: Evolução da doença e cuidados paliativos}

Os cuidadores e familiares são fundamentais para a qualidade de vida dos pacientes que recebem cuidados paliativos a nível domiciliar. Contudo, tais cuidadores também são afetados pelo processo de adoecimento devido à assistência integral oferecida aos pacientes. Essa carga pode ser de caráter físico, psicológico e social (ULLRICH A, et al., 2017).

O cuidador modifica sua vida para conviver com o paciente que possui alguma enfermidade/doença e as complicações advindas desse processo, levando-o a abdicar-se de suas vontades pessoais, passando a viver exclusivamente para atender às necessidades do paciente/ familiar. A separação entre a vida do paciente e 
do cuidador desaparece, já que o mesmo deixa de atender suas necessidades e vontades para garantir e suprir as necessidades de seu familiar (OLIVEIRA MBP, et al., 2017).

O cuidado paliativo é iniciado após o diagnóstico do paciente e intensificando-se na medida da necessidade. Consiste num direito do ser humano de ser apoiado e assistido no processo de doença até a fase final da vida. Sendo assim, integram os aspectos psicossociais e espirituais nos cuidados ao paciente, oferecendo um sistema de apoio e ajuda aos mesmos, para que vivam tão ativamente quanto possível até o processo de morte. Disponibiliza ainda, um sistema de apoio para auxiliar o paciente e sua família a lidar com a situação durante a doença e no processo de luto. Exigem uma abordagem em equipe multidisciplinar, dando continuidade à atenção até o desfecho da necessidade de sua permanência na dinâmica familiar (MENDES EC e VASCONCELLOS LCF, 2015).

Os cuidados paliativos fornecidos em domicílio do paciente seguem de acordo com a evolução das necessidades do enfermo. Tanto a doença quanto o tratamento causam impacto não somente na vida do paciente, mas também da sua família e do cuidador. Por isso, é preciso que se estabeleça uma dinâmica para que a família compreenda a evolução da enfermidade, suas limitações e as mudanças na qualidade de vida e vulnerabilidade, não somente do doente, mas também do cuidador (CUNHA AS, et al., 2018).

Com o avanço da doença, há um aumento da necessidade de cuidados oferecidos ao doente, o que exige dos cuidadores maior dedicação, trazendo mais cansaço e desgaste, visto que os pacientes requerem uma atenção no que tange ao controle da dor, do sofrimento e aos cuidados psicológicos que são necessários para amenizar estes sintomas. A dedicação será exigida por longos períodos de tempo conforme a dependência da assistência aumenta. Oferecer esses cuidados de maneira ininterrupta traz uma sobrecarga emocional, física, social, resultando em alterações psicossomáticas ao cuidador, comprometendo sua qualidade de vida e sua saúde (PRATA HM, 2017; ROCHA EM, et al., 2020).

\section{Carga emocional dos cuidadores}

Os cuidadores são vistos não somente como pessoas que auxiliam nos momentos finais dos pacientes, mas também como unidades de cuidado. É preciso que haja uma integração entre o cuidado prestado ao paciente por seu cuidador e o suporte para manutenção da sua saúde física e mental de seus familiares (LEROY T, et al., 2016).

De acordo com Traub L (2016), o cuidador familiar do paciente paliativo, o acompanha pelas etapas: diagnóstico, tratamento, reabilitação, cuidados paliativos e óbito, o que se torna uma tarefa desgastante para o cuidador, afastando-o de suas atividades rotineiras, acarretando gastos financeiros, privação do convívio social e íntimo contato com dor e sofrimento. Segundo Franchini L, et al. (2020) os cuidadores nem sempre demonstram seu sofrimento ou reclamam da carga de cuidado, preferem focar na condição do paciente e salientam-se de preocupação, especialmente com a evolução da doença e dos sintomas.

O familiar/cuidador exercem funções desconhecidas e que muitas das vezes exigem técnicas para a elaboração como: realização de curativos, higiene, administração de algumas medicações, manejo de sondas e drenos, manejo de aparelhos como pressão positiva contínua das vias aéreas (CPAP) respiradores/aspiradores, manutenção de vias aéreas, além de lidar com agravamento do quadro de saúde daquele familiar e a possibilidade de morte. Essa demanda leva em consideração que o atendimento domiciliar é especial e trabalhoso, principalmente nas fases tardias da doença. Portanto, essas atividades levam o familiar/cuidador a um estado de estresse emocional, desgaste físico, social e econômico, o que afetará sua qualidade de vida globalmente (OLIVEIRA MBP, et al., 2017).

A sobrecarga do cuidador pode ser compreendida como um conjunto de fatores que influenciam na piora da sua qualidade de vida devido ao contato próximo e por longo período de tempo com o paciente em cuidados paliativos. São as consequências negativas que levam o cuidador a algum tipo de comprometimento, seja econômico, psicológico, físico ou emocional. É importante implementar estratégias que previnam esse tipo de consequência para que não haja piora na qualidade de vida deste, pois estes problemas, ao piorar a qualidade de vida do cuidador, também pode influenciar negativamente os cuidados oferecidos ao enfermo e até mesmo estender o período de luto dos cuidadores após a morte do doente (PRATA HM, 2017). 


\section{Processo de luto vivenciado pelos cuidadores}

Quando o paciente se encontra em estado terminal, tendem a aumentar todos os fatores estressantes, isto devido à maior intensidade dos sintomas manifestados pelo doente. É importante que os profissionais de saúde saibam que estas complicações são tratáveis e, para tanto, precisam estar qualificados e capacitados para identificá-las e intervir de modo apropriado (TRAUB L, 2016).

Ainda segundo Traub L (2016) o fortalecimento de vínculos familiares a partir do adoecimento, e o contato com a possibilidade da morte contribuíram para a valorização das relações, visto, como mudanças benéficas na vida desses e dos pacientes. Sendo que mudanças físicas e psíquicas após o câncer também podem representar a possibilidade de uma nova vida para ao paciente e sua família.

De acordo com Duimering A, et al. (2020) o desgaste emocional causa mais sofrimento do que os cuidados físicos e conforme a doença avança, deficiências, progressão dos sintomas e luto antecipatório causam sofrimento significativo aos cuidadores.

Mesmo diante todo esgotamento físico e mental percebido nos cuidadores, alguns expressam a vontade incessante de permanecerem próximo ao paciente até o último momento de vida, como se isso oferecesse ao cuidador um tipo de fechamento à trajetória da assistência. Podendo observar que, a partir do momento em que há consciência por parte dos familiares que o fim da vida está mais próximo, estes tendem a permanecer junto ao doente a maior parte do tempo possível (CORREIA IMTB e TORRES GV, 2011).

Ao promover a morte digna o cuidador tem a capacidade de oferecer acolhimento, amparo e auxílio ao doente e sua família. Essa característica está associada em oferecer uma assistência adequada aos envolvidos nesse momento difícil. Portanto, deve ser promovida uma assistência ampla, considerando-se os aspectos psicológicos que envolvem uma doença sem possibilidade de cura em estágio avançado. É de responsabilidade da equipe multidisciplinar, em especial, ao médico, atuar com sensibilidade e eficácia, tirando as dúvidas e encorajando atitudes positivas durante o processo de terminalidade e de luto (FERNANDES MA, et al., 2015).

Estudos, segundo Delalibera M, et al. (2015), evidenciaram que os cuidadores que apresentavam maior nível de sobrecarga durante a fase de prestação de cuidados ao paciente estavam mais propensos a desenvolver um luto complicado, prolongado ou traumático onde a sobrecarga no cuidar é um dos fatores de risco para o desenvolvimento de complicações no luto. Em pesquisas anteriores foi mostrado que o apoio psicológico prestado aos cuidadores os ajuda a obter resultados de luto mais favoráveis, com risco diminuído de luto complicado.

\section{Família dos pacientes na função de cuidadores: desafios e ponto de vista}

Os familiares na função de cuidadores, em grande parte, não se sentem confiantes em prestar o atendimento ao seu familiar na posição de paciente, ficando inseguros em prestar as atividades necessárias, visto que não foram preparados para desenvolver essa função. $E$ na grande maioria dos casos, o ato de cuidar não surge como obrigação, mas como uma escolha (BLANC LO, et al., 2016).

O familiar na função de cuidador desenvolve as responsabilidades necessárias frente às responsabilidades pelos cuidados, e enquanto um outro grupo familiar muitas das vezes assume seu papel de forma indireta, não estando relacionado ao cuidado, sobrecarregando aquele cuidador tanto psicologicamente quanto fisicamente. A importância de se ter acompanhamento com equipe multidisciplinar principalmente especializada com cuidados paliativos auxilia o cuidador e toda família no processo do cuidado com a doença daquele enfermo. As orientações/ informações repassadas pela equipe contribuem tanto para o paciente quanto ao seu cuidador, trazendo mais confiança, conhecimento e capacidade de realizar as atividades/ técnicas necessárias no dia a dia.(OLIVEIRA MBP, et al., 2017).

\section{Religiosidade dos cuidadores}

Sendo a fé utilizada como uma das principais estratégias para suportar o sofrimento, através dela o cuidador e paciente se fortalecem para buscar o conforto e descanso necessários. Desta forma o cuidador passa a observar os cuidados como uma forma de plantio para colheita futura (OLIVEIRA MBP, et al., 2017). 
Segundo, Cervelin AF e Kruse MHL (2014) os valores religiosos devem ser respeitados, devendo o cuidador incentivar a participação do paciente e familiares na prática religiosa, mas que não deve ser imposta. É de suma importância saber ouvir e respeitar as diferenças religiosas entre doente e responsável pela assistência, cabendo ao mesmo a coleta de informação sobre o assunto no início do tratamento, tornando-a rotineira. O uso da fé e a crença em Deus fornecem ao cuidador esperança e confiança para lidar com os obstáculos emocionais e físicos apresentados pelo paciente, sendo argumentos que justificam a abordagem espiritual/ religiosa e são relevantes durante o tratamento paliativo.

A fé, a espiritualidade e as práticas religiosas são percebidas pelos cuidadores como método eficiente de encarar a sobrecarga da responsabilidade, da angústia, do estresse e da depressão decorrentes do processo de cuidar, facilitam a luta nas dificuldades, dão sentido à vida, à velhice, à dependência e ao cuidar. Favorece a interpretação dos eventos de forma mais positiva, encarando de forma mais compreensível o tratamento (ARAÚJO JA e LEITÃO EMP, 2012).

\section{Sentimentos dos cuidadores}

Estudos apontam que ocorrem problemas de saúde com muita frequência nos cuidadores de pacientes que recebem cuidados paliativos em casa. Há relatos de ocorrência de distúrbios de sono, exaustão física e emocional, isolamento social, problemas financeiros, alto nível de ansiedade, angústia, transtorno de estresse pós-traumático (TEPT), depressão e problemas emocionais como incertezas e desesperanças (KIM SH, et al., 2015).

Alguns autores apontam que a carga sobre os cuidadores aumenta progressivamente conforme a progressão da doença, provocando o aumento da ansiedade. Os cuidadores possuem necessidades não somente com relação ao planejamento e cuidados ao paciente, mas também sobre o gerenciamento do estado físico do paciente, o aumento das limitações, a aproximação do paciente da sua morte e a necessidade de viver o momento de luto (KRUG K, et al., 2016).

O apoio aos familiares merece uma atenção especial, visto que as psicoterapias têm como foco o paciente e seus resultados de melhora e, quase sempre, não abordam familiares, amigos e cuidadores. A Organização Mundial da Saúde em sua definição de cuidados paliativos defende que todos os aspectos do cuidado devem ser abordados, inclusive os aspectos psicossociais. Melhorando o suporte familiar, há melhora na experiência de final de vida dos pacientes. Apontada em alguns estudos, a terapia da dignidade parece exercer uma boa influência no variável suporte familiar, podendo assim, ser a terapia de escolha para melhorar esses aspectos (DONATO SCT, et al., 2016).

Com base nessa constatação, considera-se que oferecer cuidados ao paciente em fase avançada de enfermidade influencia diretamente na qualidade de vida do cuidador, pois o conjunto de aspectos físicos, emocionais, sociais e econômicos sofrem reflexo de sua dedicação e cuidado ao enfermo. $O$ fato do paciente necessitar de cuidados completos, principalmente nos seus momentos finais de vida, faz com que o cuidador se sinta parte do processo de adoecimento e de perda (REZENDE G, 2017).

\section{O desgaste físico do cuidador/ familiar em função da assistência aos doentes em cuidados paliativos}

A sobrecarga física, fica evidente pelo esgotamento físico, decorrente das ações de empenho, e as dificuldades de cuidar no domicílio. O esforço contínuo requerido no encargo, juntamente com a ocupação seja social e/ou familiar são fatores que resultam com prejuízo na saúde física do cuidador. A progressão da debilidade física do paciente em virtude do avanço da doença, faz com que o responsável pela assistência assuma de forma integral todas as necessidades, sejam fisiológicas ou de conforto, fazendo com que esses cuidados diários, que no início são bem tolerados, mas a longo prazo, pesam ao cuidador, gerando um desgaste físico (MOREIRA RS e TURRINI RNT, 2011).

De acordo com Araújo JA e Leitão EMP (2012), os estudos relacionados a essa temática, apresentam trabalhos significativos relacionando a degeneração da saúde e adoecimento do cuidador, tanto no contexto físico e psicossocial, que com o passar do tempo afetam diretamente o contexto familiar, por diminuir o poder aquisitivo, romper vínculos, aumenta 0 isolamento e o estresse, gerando insatisfação com a vida e 0 aparecimento de morbidades. 
Vale destacar que relacionar dor, vitalidade e aspectos físicos com a tarefa de cuidar, dá uma dimensão de alguns sintomas relatados como a baixa energia e vigor, falta de vontade, fadiga, esgotamento, dor e cansaço podem ser consequências do trabalho excessivo (OLIVEIRA DC, et al., 2011).

\section{CONSIDERAÇÕES FINAIS}

A partir desta revisão, conclui-se que os pacientes que se encontram em cuidados paliativos, necessitam de acompanhamento em tempo integral, sendo os cuidadores os responsáveis por oferecer todo o suporte emocional, financeiro e atender as necessidades pessoais destes pacientes. Na realidade, devido a esses cuidados excessivos e dedicação em tempo integral, os cuidadores ficam sobrecarregados e desgastados tanto fisicamente quanto emocionalmente, comprometendo sua qualidade de vida. Desta forma, é preciso que se realize um trabalho preventivo e de suporte com estratégias que visem proporcionar atenção à saúde, como atendimentos médicos e psicológicos, para que possam reduzir os impactos sobre a vida destes cuidadores.

\section{REFERÊNCIAS}

1. ARAÚJO JA, LEITÃO CMP. O Cuidador do paciente em cuidado paliativo: sobrecarga e desafios. Revista do Hospital Universitário Pedro Ernesto, UERJ - 2012; Ano 11.

2. BLANC LO, et al. Compreendendo as experiências vividas pelos familiares cuidadores frente ao paciente oncológico. Pensando Famílias, 2016; 20(2): 132-148.

3. CERVELIN AF, KRUSE MHL. Espiritualidade e religiosidade nos cuidados paliativos: conhecer para governar. Escola Anna Nery Revista de Enfermagem, 2014; 18(1): 136- 142

4. CORREIA IMTB, TORRES GV. The familycaregiver in the face ofthesicknear death oncologicalendoflife. Rev. enferm. UFPE online. $2011 ; 5$ (spe): 399-409.

5. CUNHA AS, et al. Cuidado paliativo oncológico: percepção dos cuidadores. J. Health BiolSci. 2018; 6(4): 383-390.

6. DELALIBERA M, et al. Sobrecarga no cuidar e suas repercussões nos cuidadores de pacientes em fim de vida: revisão sistemática da literatura. Ciência \& Saúde Coletiva, 2015; 20(9): 2731-2747.

7. DONATO SCT, et al. Efeitos da terapia da dignidade em pacientes terminais: uma revisão sistemática. Revista da Escola de Enfermagem da USP, 2016; 50(6): 1014-1024.

8. DUIMERING A, et al. Informal caregiver quality of life in a palliative oncology population. Support Care Cancer, 2020; 28: 1695-1702.

9. FERNANDES MA, et al. Cuidados paliativos e luto: um estudo bibliométrico - Esc Anna Nery 2016; 20 (4): e20160102.

10. FERNANDES MA, et al. Cuidados paliativos e luto: compreensão de médicos residentes - Revista de Pesquisa Cuidado é Fundamental Online. J. res.: fundam. care. online 2015; ISSN 2175-5361.

11. FRANCHINI I, et al. Caregivers in home palliative care: gender, psychological aspects, and patient's functional status as main predictors for their quality of life. Support Care Cancer 2020; 28(3227-3235).

12. HERMES HR, LAMARCA ICA. Cuidados paliativos: uma abordagem a partir das categorias profissionais de saúde. Ciênc. Saúde coletiva, 2013; 18: 9.

13. KIM SH, et al. Associação entre o estado emocional de cuidadores familiares e o tempo de permanência em uma unidade de cuidados paliativos: um estudo retrospectivo. Cuidados de suporte Palliat. 2015; 13: 1695 - 700.

14. KOHLER LB, et al. Cuidados paliativos ambulatoriais e qualidade de vida em pacientes oncológicos. Diagn Tratamento. 2016; 21(3): 101-5.

15. KRUG K, et al. Correlação entre a qualidade de vida do paciente em cuidados paliativos e a sobrecarga de seus cuidadores familiares: um estudo de coorte observacional prospectivo. BMC PalliatCare. 2016; $15: 4$.

16. LACERDA MS, et al. Depressivesymptoms in thefamilycaregiversofpatientswithheartfailure: anintegrative review. Rev Gaúcha Enferm. 2019; 40: e201800571.

17. LEROY T, et al. Visões cruzadas de sobrecarga e sofrimento emocional de pacientes com câncer e cuidadores familiares durante os cuidados paliativos. Psicooncologia. 2016; 25: $1278-85$.

18. MENDES EC, VASCONCELOS LFC. Cuidados paliativos no câncer e os princípios doutrinários do SUS, 2015 ; 39(106): 881-892.

19. MOREIRA RS, TURRINI RNT, Paciente oncológico terminal: sobrecarga do cuidador. Revista eletrônica trimestral de Enfermería, 2011; 22: 4-5.

20. OLIVEIRA DC, et al. Qualidade de vida e sobrecarga de trabalho de cuidadores de idosos em seguimento ambulatorial. Texto Contexto Enferm, Florianópolis, 2011; 20(2): 234-40.

21. OLIVEIRA MBP, et al. Assistência domiciliar oncológica: percepção da família e do cuidador sobre os cuidados paliativos. Escola Anna Nery, 2017; 21(2): e20170030.

22. PEREIRA CR, et al. A espiritualidade enquanto estratégia de enfrentamento para o cuidador familiar frente à terminalidade. Revista Nupem, 2020; 12(25): 125-127.

23. PINHEIRO MLA, et al. Paciente oncológico em cuidados paliativos: a perspectiva do familiar cuidador. Revenferm UFPE online., 2016; 10(5): 1749-5. 
24. PRATA HM. Cuidados paliativos e direito do paciente terminal. SP: Editora Manole 2017, p. 97.

25. PRUDENTE COM, et al. Qualidade de vida de cuidadores familiares de adulto com lesão medular: uma revisão sistemática. Ciência \& Saúde Coletiva, 2017; 22(1).

26. REZENDE G. Sobrecarga de cuidadores familiares de idosos em cuidados paliativos. 2016. 93 f. Tese (Doutorado em Ciências) - Programa de Pós-Graduação Enfermagem em Saúde Pública, Universidade de São Paulo, São Paulo, 2016.

27. ROCHA EM, et al. Sobrecarga do cuidador de pacientes oncológicos em cuidados paliativos. Rev enferm UFPE online. 2020; 14: e244165.

28. ROCHA EM, SOUZA A. Sobrecarga do cuidador e o impacto na qualidade de vida dos pacientes oncológicos em cuidados paliativos. SEFIC Unilasalle 2017.

29. TRAUB L. Cuidador de familiar com câncer: papéis e necessidades. Psicologia.pt 2016; ISSN 1646-6977.

30. ULLRICH A, et al. Quality of life, psychological burden, needs, and satisfaction during specialized in patient palliative care in Family care givers of advanced cancerpatient. BMC Palliative Care, 2017; 16:31. 